\title{
Editorial: Symbiotic Relationships as Shapers of Biodiversity
}

\author{
Guillaume Chomicki ${ }^{1 *}$, Roxanne Beinart ${ }^{2}$, Carlos Prada ${ }^{3}$, Kimberly B. Ritchie ${ }^{4}$ and \\ Marjorie Gail Weber ${ }^{5}$ \\ ${ }^{1}$ Ecology and Evolutionary Biology, School of Biological Sciences, The University of Sheffield, Sheffield, United Kingdom, \\ ${ }^{2}$ Graduate School of Oceanography, University of Rhode Island, Narragansett, RI, United States, ${ }^{3}$ Department of Biological \\ Sciences, University of Rhode Island, Kingston, Rl, United States, ${ }^{4}$ Department of Natural Sciences, University of South \\ Carolina Beaufort, Bluftton, SC, United States, ${ }^{5}$ Program in Ecology, Evolution, and Behavior, Department of Plant Biology, \\ Michigan State University, East Lansing, MI, United States
}

Keywords: symbiosis, biodiversity, climate change, microbes, coral

\section{Editorial on the Research Topic}

\section{Symbiotic Relationships as Shapers of Biodiversity}

Symbiosis - the living together of unlike organisms (de Bary, 1878) - is exceedingly common in nature. Symbiotic relationships modify the physiology and influence the ecological dynamics and evolutionary processes of interacting partners, ultimately altering the distribution of species across the planet (Douglas, 2010; Chomicki et al., 2019). This is particularly true with microbial symbiosis. To date, research has demonstrated that symbionts play a pivotal role in shaping biodiversity at ecological and evolutionary scales. However, many facets of the relationship between symbiosis and the generation and maintenance of biodiversity remain unexplored. In the face of unprecedented climatic changes, biodiversity studies incorporating symbiotic relationships will be key to understanding how species will endure changing environmental conditions.

In this Research Topic, we have assembled a collection of articles that broaden our understanding of how symbiotic relationships between hosts and microbes alter biodiversity patterns at ecological and evolutionary scales and as a result of environmental change.

PU), United States

${ }^{*}$ Correspondence:

Guillaume Chomicki g.chomicki@sheffield.ac.uk

Specialty section:

This article was submitted to Coevolution,

a section of the journal

Frontiers in Ecology and Evolution

Received: 07 January 2022

Accepted: 24 January 2022

Published: 16 February 2022

Citation:

Chomicki G, Beinart R, Prada C

Ritchie KB and Weber MG (2022)

Editorial: Symbiotic Relationships as

Shapers of Biodiversity.

Front. Ecol. Evol. 10:850572.

doi: 10.3389/fevo.2022.850572

\section{SYMBIOSES MODULATE RESPONSES TO ENVIRONMENTAL CHANGE}

This Research Topic addresses how and when symbioses shape species' responses to environmental changes using a variety of systems and approaches.

Maher et al. showed that coral microbiomes exhibit flexibility and resilience following thermal stress. The authors performed an experiment during a 2016 heat stress event on Moorea Island, testing how heat stress, with the addition of two forms of nitrogen, affects the coral microbiome. The authors found that heat stress overwhelms the system, masking the effect of nitrogen. Following heat stress, the coral microbiome became dominated by a bacterial clade known as Endozoicomonas, a signature that persisted following the heat stress event. Because Endozoicomonas is a likely beneficial symbiont, thermal stress-mediated remodeling of the coral microbiome may play a key role in coral reef resilience to climate change.

Fuess et al. highlighted how an increase in algal symbiont density, as a result of nitrogen water pollution, drives an increased coral host susceptibility to disease via a reduced immunity. Thus, low water quality can increase coral mortality indirectly, by driving up symbiont density which in turn reduces the expression of immunity genes. 
Thurber et al. reviewed the state-of-the-art in coral disease dynamics. They argued that disease outbreaks are largely driven by changing environments, and suggest an integrative approach that includes evaluating the impacts of changing environments on the host, its microbiome, and the environment in tandem.

Nourabadi and Nichiguchi asked how a fluctuating environment affects the recruitment and success of environmentally-acquired symbionts, using the symbiosis between Hawaiian bobtail squids and their bioluminescent bacterial symbionts. Using experimental evolution, they showed that Vibrio strains adapted to low $\mathrm{pH}$ have both more efficient colonization rate and higher competence (bioluminescence) than ancestral strains. Their results suggest that environmental change can drastically affect the functioning of laterally-acquired symbioses.

Soto and Nishiguchi reviewed the current understanding of how environmental stress drives Vibrio symbiont diversity. The authors discuss the mechanisms enabling Vibrio to cope with environmental stress in both their free-living and symbiotic stages, and how this in turn affects their diversity.

Work by van't Padje et al. tested how mycorrhizal symbioses are affected by extreme weather events. In particular, they tested how phosphorus is up-taken and transferred to the plant host under extreme heat stress or flooding-relying on a recently developed technique based on quantum-dot tagging of nutrients, which allows to track nutrients along the fungal network using fluorescence (Whiteside et al., 2019). Phosphorus transfer, as well as plant and fungal survival, were deeply affected by flooding, but less so by heat treatment. Their innovative approach opens new avenues to test how different plant-host combinations are impacted by extreme weather events.

\section{SPECIFICITY AND DEPENDENCE AS KEY FACTORS OF SYMBIOTIC RELATIONSHIPS}

The level of dependence (facultative to obligate) and specialization/specificity (generalist: many partners to specialist: few specific partners) is pivotal in driving stability of symbiotic mutualisms across ecological and evolutionary scales (Chomicki et al., 2020). Contribution to this Research Topic demonstrates how specificity and dependence are key to predict species' response to climate change, pinpointing a number of essential areas for future research.

Ginete and Goodrich-Blair reviewed state-of-the-art research on the specificity of host-symbiont systems. They argue that recognizing below species level strain variation is critical to understand symbiotic specificity. The authors recognized two main factors driving specificity: (1) factors that contribute a required activity in support of the association and (2) factors involved in antagonistic interactions with organisms outside of the association. Identifying these factors across host-symbiont partnerships is the key understanding the drivers of specificity in microbial symbioses.

Goulet and Goulet reviewed current data on dependence and specificity in the large array of Cnidarian symbioses from coral reefs. Because many Cnidarian symbioses are obligate and show high specificity, they are likely to be strongly impacted by the effects of climate change. Thus, the robustness and potential plasticity of the host-symbiont association will determine the survival of the symbioses following environmental change. Understanding the dependence, specificity as well as performance and plasticity of host symbiont combinations in Cnidarian symbiosis is therefore critical to forecast the effect of climate change on these extremely biodiverse communities.

\section{SYMBIOSES AS DRIVERS OF ECOLOGICAL COMMUNITY DYNAMICS}

Microbial symbioses are ubiquitous interactions in nature. As such, they play key roles in structuring and modulating ecological communities. Contributions to this Research Topic show how symbiosis can drive species composition within communities, but also how it can promote species coexistence. This in turn, can feed back on the functioning of the symbiosis.

Sanders-Smith et al. studied the leaf surface microbiome of the seagrass Zostera marina, a foundation species which sustains key temperate marine ecosystems in British Columbia, Canada. The authors found distinct microbiome communities on young and old leaves. Older leaves had richer leaf microbiomes and showed more geographic microbiome differentiation a, suggesting host filtering and environmental influence, with core microbial taxa suggesting functional roles. Understanding how the leaf microbiome affects the growth, epiphyte control, and detoxification of seagrass waste product is key to unlocking the factors maintaining complex marine ecosystems.

Herder et al. asked how the interplay of intrinsic host vs. extrinsic environmental factors shapes the fecal microbiome, using Anna's hummingbird as a model system. They found that the greatest explanatory variable was intrinsic, but the environment had a substantial effect on the abundance of bacterial lineages. The authors argue that understanding how the environment shapes microbiome-host symbiosis has profound consequences for species conservation.

Runte et al. investigated how symbiosis predicts fungal communities in mixed conifer-hardwood communities. They found that the turnover of ectomycorrhizal and saprotrophic communities was differentially predicted by the availability of canopy species, though both were driven by similar soil properties. At the study scale, rRNA was a much better predictor than DNA. This work reveals the importance of ectomycorrhizal symbiosis in structuring soil fungal communities.

Hsu and Moeller tested whether the metabolic symbiosis between Paramecium bursaria and the endosymbiotic green algae Chlorella impacts species coexistence. Specifically, they tested whether the symbiosis with Chlorella influences the coexistence of P. bursaria and its non-symbiotic competitor Colpidium. Their study reveals light-dependent priority effects modulating competition between both bacterivorous species, which pinpoints the pivotal role that mutualisms can play as modulators of species coexistence (Chomicki et al., 2019).

Leclair et al. tested whether the endosymbiont communities of different pea aphid biotypes had a protective effect on their 
hosts under natural conditions and whether endosymbiotic associations and parasitoid communities associated with the pea aphid complex were linked. The authors confirmed the protective effects of endosymbionts on their hosts in nature, and found a positive correlation between the $\alpha$ and $\beta$ diversities of the endosymbiont and parasitoid communities, indicating interactions between these two guilds. Their work emphasizes the importance of field studies of symbioses.

\section{OUTLOOK}

Altogether, this Research Topic broadens our understanding of the diverse ways in which microbial symbiosis can alter and be altered by biodiversity changes across scales. It pinpoints the need to give a central place to symbiotic partnerships in the study of species response to climate change. It also highlights

\section{REFERENCES}

Chomicki, G., Kiers, E. T., and Renner, S. S. (2020). The evolution of mutualistic dependence. Ann. Rev. Ecol. Evol. Syst. 51, 409-432. doi: 10.1146/annurev-ecolsys-110218-024629

Chomicki, G., Weber, M., Antonelli, A., Bascompte, J., and Kiers, E. T. (2019). The impact of mutualisms on species richness. Trends Ecol. Evol. 34, 698-711. doi: 10.1016/j.tree.2019.0 3.003

de Bary (1878). Ueber symbiose. Tageblatt für die Versammlung deutscher Naturforscher und Aerzte 51, 121-126.

Douglas, A. E. (2010). The Symbiotic Habit. Princeton, N.J.: Princeton University Press.

Whiteside, M. D., Werner, G. D., Caldas, V. E., van't Padje, A., Dupin, S. E., Elbers, B., et al. (2019). Mycorrhizal fungi respond to resource inequality by moving phosphorus from rich to poor patches across networks. Curr. Biol. 29, 2043-2050. doi: 10.1016/j.cub.2019.0 4.061 several future research avenues aiming at understanding how symbiotic associations shape-and are shaped by-biodiversity.

\section{AUTHOR CONTRIBUTIONS}

GC wrote first draft with edits from RB, CP, KR, and MW. All authors contributed to the article and approved the submitted version.

\section{FUNDING}

GC is funded by the Natural Environment Research Council (NE/S014470/2). CP has been supported with NSF-OIA 2032919 and USDA-NIFA 1017848.

Conflict of Interest: The authors declare that the research was conducted in the absence of any commercial or financial relationships that could be construed as a potential conflict of interest.

Publisher's Note: All claims expressed in this article are solely those of the authors and do not necessarily represent those of their affiliated organizations, or those of the publisher, the editors and the reviewers. Any product that may be evaluated in this article, or claim that may be made by its manufacturer, is not guaranteed or endorsed by the publisher.

Copyright (C) 2022 Chomicki, Beinart, Prada, Ritchie and Weber. This is an openaccess article distributed under the terms of the Creative Commons Attribution License (CC BY). The use, distribution or reproduction in other forums is permitted, provided the original author(s) and the copyright owner(s) are credited and that the original publication in this journal is cited, in accordance with accepted academic practice. No use, distribution or reproduction is permitted which does not comply with these terms. 